\title{
Astrophysical tests of atomic data important for the stellar Mg abundance determinations
}

\author{
L. Mashonkina ${ }^{1,2}$ \\ 1 Universitäts-Sternwarte München, Scheinerstr. 1, 81679 München, Germany \\ e-mail: lyuda@usm.lmu.de; lima@inasan.ru \\ 2 Institute of Astronomy, Russian Academy of Sciences, 119017 Moscow, Russia
}

Received 19 November 2012 / Accepted 11 December 2012

\section{ABSTRACT}

\begin{abstract}
Context. Magnesium abundances of cool stars with different metallicities are important for understanding the galactic chemical evolution.

Aims. This study tests atomic data used in stellar magnesium abundance analyses.

Methods. We evaluate nonlocal thermodynamical equilibrium (NLTE) line formation for Mg I, using the most up-to-date theoretical and experimental atomic data available so far, and check the $\mathrm{Mg}$ abundances from individual lines in the Sun, four well studied A-type stars, and three reference metal-poor stars.

Results. With the adopted $g f$-values, NLTE abundances derived from the $\mathrm{Mg}$ I $4703 \AA$, $5528 \AA$, and $\mathrm{Mg}$ Ib lines are consistent within 0.05 dex for each A-type star. The same four Mg I lines in the solar spectrum give consistent NLTE abundances at $\log N_{\mathrm{Mg}} / N_{\mathrm{H}}=-4.45$, when correcting van der Waals damping constants inferred from the perturbation theory. Inelastic $\mathrm{Mg}+\mathrm{H}$ collisions as treated by Barklem, Belyaev, Spielfiedel, Guitou, and Feautrier serve as an efficient thermalizing process for the statistical equilibrium of Mg I in the atmospheres of metal-poor stars. The use of $\mathrm{Mg}+\mathrm{H}$ collision data improves $\mathrm{Mg}$ abundance determinations for $\mathrm{HD} 84937$ and HD 122563, though it does not completely remove the differences between different lines.
\end{abstract}

Key words. atomic data - atomic processes - line: formation - Sun: abundances - stars: abundances - stars: atmospheres

\section{Introduction}

Magnesium plays an important role in studies of cool stars. It affects atmospheric structure by donating free electrons and with a significant contribution of $\mathrm{Mg}$ I photoionization to the ultraviolet (UV) opacity. Magnesium is a key element for studying the history of $\alpha$-process nucleosynthesis in the Universe. $\mathrm{The} \mathrm{Mg} / \mathrm{Fe}$ abundance ratio of metal-poor (MP) stars carries information on the initial mass function and star formation rate in our Galaxy, dwarf galaxies of the Local Group, and distant galaxies (see Tolstoy et al. 2009, for references). Magnesium is one of the best observed elements in A to late-type stars, though it is represented by only a few lines of neutral atoms in the visual spectral range. The $\mathrm{Mg} \mathrm{Ib}$ lines at 5167, 5172, and $5183 \AA$ can be measured over a wide range of metallicity from super-solar values down to $[\mathrm{Fe} / \mathrm{H}]^{1}=-5.5$ (Frebel et al. 2005) and over a wide range of effective temperature from $3000 \mathrm{~K}$ up to $12000-13000 \mathrm{~K}$ (Przybilla et al. 2001). In stellar atmospheres with $T_{\text {eff }}>4500 \mathrm{~K}$, neutral magnesium is a minority species, and its statistical equilibrium (SE) can easily deviate from thermodynamic equilibrium due to deviations in the mean intensity of ionizing radiation from the Planck function. Since the end of the 1960s, the problem of nonlocal thermodynamic equilibrium (NLTE) line formation for $\mathrm{Mg} \mathrm{I}$ in stellar atmospheres was considered in many studies. The model atoms of Mg I have been created by Athay \& Canfield (1969), Lemke \& Holweger (1987), Mauas et al. (1988), Gigas (1988), Carlsson et al. (1992), Mashonkina et al. (1996), Zhao et al. (1998),

\footnotetext{
1 In the classical notation, where $[\mathrm{X} / \mathrm{H}]=\log \left(N_{\mathrm{X}} / N_{\mathrm{H}}\right)_{\text {star }}-$ $\log \left(N_{\mathrm{X}} / N_{\mathrm{H}}\right)_{\text {Sun }}$.
}

Gratton et al. (1999), Idiart \& Thévenin (2000), Przybilla et al. (2001), Mishenina et al. (2004), and Merle et al. (2011).

This study was motivated by the recent detailed quantum mechanical calculations of Barklem et al. (2012, hereafter, BBSGF) for inelastic $\mathrm{Mg}+\mathrm{H}$ collisions. The role of inelastic collisions with neutral hydrogen atoms in establishing the SE of atoms in cool stars has been debated for decades. Steenbock \& Holweger (1984) implemented the classical Drawin $(1968,1969)$ formula to calculate H I collision rates, and it suggests that their influence is comparable to electron impacts. Up recent times, laboratory measurements and/or detailed quantum mechanical calculations of collisions with $\mathrm{HI}$ atoms have only been available for $\mathrm{Na}$ I and Li I (see Barklem et al. 2011, for references). Based on these data, the Drawin formalism has been criticized for not providing a realistic description of the physics involved and overestimating the collision rates. Interestingly, spectroscopic studies of Na I also suggest that Drawin's formula strongly overestimates the $\mathrm{Na}+\mathrm{H}$ collision rates. For example, Allende Prieto et al. (2004) has reproduced the center-to-limb variation of the solar Na I $6160 \AA$ line with pure electronic collisions. At the same time, the need for a thermalizing process not involving electrons in the atmospheres of, in particular, very metal-poor stars, has been indicated by many NLTE line-formation studies (see Mashonkina et al. 2011, and references therein).

The main goal of this paper is to investigate how the use of recent data of BBSGF on $\mathrm{Mg}+\mathrm{H}$ collisions influences the NLTE results for $\mathrm{Mg}$ I. We started from an analysis of the solar $\mathrm{Mg}$ I lines, for which the departures from LTE are small, and the derived abundances are not influenced by $\mathrm{H}$ I collision treatment. It was found that the two lines Mg I $4703 \AA$ and $\operatorname{Mg}$ I $5528 \AA$ 
give significantly lower abundance compared with that from the $\mathrm{Mg} \mathrm{Ib}$ lines and also compared with the meteoritic value. To understand the source of discrepancies and to differentiate the effects of oscillator strengths and van der Waals broadening, we moved to the hotter A-type stars.

The paper is organized as follows. All our results are based on the NLTE line formation for Mg I. The model atom of magnesium and the used atomic data are described in Sect. 2. In Sect. 3, we derive abundances from individual $\mathrm{Mg}$ I lines in the solar spectrum and in the spectra of four well studied A-type stars. Section 4 investigates the effect of $\mathrm{Mg}+\mathrm{H}$ collisions on the SE of magnesium and NLTE abundances for the two very metal-poor $([\mathrm{Fe} / \mathrm{H}]<-2$, VMP) stars. We compare the NLTE results from calculations using $\mathrm{Mg}+\mathrm{H}$ collision data of BBSGF and using the classical Drawinian rates. The NLTE abundance corrections are presented for lines of $\mathrm{Mg} \mathrm{I}$ in the grid of metal-poor model atmospheres. Our conclusions are given in Sect. 5.

\section{Method of NLTE calculations for magnesium}

This study uses a comprehensive model atom that includes 85 levels of $\mathrm{Mg}$ I, 2 levels of $\mathrm{Mg}$ II, and the ground state of $\mathrm{Mg}$ III. For Mg I, we rely on the model atom produced by Zhao et al. (1998), who carefully investigated atomic data on the energy levels, transition probabilities, and photoionization cross sections. In this study, the calculation of collisional rates was updated. For electron-impact excitation, we used rate coefficients of Mauas et al. (1988), where available, and the same recipes as in Zhao et al. (1998) for the remaining transitions. Ionization by electronic collisions was calculated from the Seaton (1962) formula with a mean Gaunt factor set equal to $\bar{g}=0.1$ for $\mathrm{Mg}$ I and to 0.2 for $\mathrm{Mg}$ II. For $\mathrm{HI}$ impact excitations and charge transfer processes $\mathrm{Mg} \mathrm{I}+\mathrm{HI} \leftrightarrow \mathrm{Mg} \mathrm{II}+\mathrm{H}^{-}$, rate coefficients were taken from the detailed quantum mechanical calculations of BBSGF.

Singly ionized magnesium is represented in our model atom by the two levels $3 s^{2} \mathrm{~S}$ and $3 p^{2} \mathrm{P}^{\circ}$. Their energies and $g f$-value of the resonance transition were taken from the NIST database $^{2}$ (Ralchenko et al. 2008). Photoionization is treated by utilizing Opacity Project cross-sections as available through the TOPBASE database ${ }^{3}$.

To solve the coupled radiative transfer and statistical equilibrium equations, we used a revised version of the DETAIL program (Butler \& Giddings 1985). The update was described by Przybilla et al. (2011). Calculations were performed with planeparallel (1D), LTE, and blanketed model atmospheres computed for given stellar parameters. These are the MAFAGS-OS models by Grupp et al. (2009) for the Sun and VMP stars, the LLMODELS models (Shulyak et al. 2004) by D. Shulyak for the three A-type stars, and the model by R. Kurucz ${ }^{4}$ for Sirius.

Table 1 lists the lines of $\mathrm{Mg}$ I used in abundance analyses for MP stars, together with the adopted line data. Oscillator strengths were taken from the laboratory measurements of Aldenius et al. (2007) for the $\mathrm{Mg}$ Ib lines, calculations of Chang \& Tang (1990) for MgI $4703 \AA$ and 5528 $\mathrm{A}$, and calculations of Tachiev \& Froese Fischer for MgI $4571 \AA$ as presented in the NIST database. An accuracy of predicted $g f$-values can only be estimated for the $\mathrm{Mg}$ Ib lines, where the difference between calculations of Chang \& Tang (1990) and measurements of Aldenius et al. (2007) amounts to 0.06 dex. The van der Waals broadening constants $\Gamma_{6}$ were computed with cross-sections

\footnotetext{
2 http://WwW.nist.gov/pml/data/asd.cfm

3 http://cdsweb.u-strasbg.fr/topbase/xsections.html

4 http://kurucz.harvard.edu/stars/SIRIUS/
}

Table 1. Mg I line data.

\begin{tabular}{lcccc}
\hline \hline $\begin{array}{l}\lambda \\
(\AA)\end{array}$ & $\begin{array}{l}E_{\text {exc }} \\
(\mathrm{eV})\end{array}$ & $\log g f$ & $\begin{array}{c}\log \Gamma_{4} / N_{\mathrm{e}} \\
\left(\mathrm{rad} / \mathrm{s} \mathrm{cm}^{3}\right)\end{array}$ & $\begin{array}{l}\log \Gamma_{6} / N_{\mathrm{H}} \\
\left(\mathrm{rad} / \mathrm{s} \mathrm{cm}^{3}\right)\end{array}$ \\
\hline 4571.10 & 0.00 & $-5.62^{1}$ & -6.46 & -7.77 \\
4702.99 & 4.33 & $-0.44^{2}$ & -4.17 & -6.69 \\
5528.41 & 4.33 & $-0.50^{2}$ & -4.63 & -6.98 \\
5172.68 & 2.71 & $-0.45^{3}$ & -5.43 & -7.27 \\
5183.60 & 2.71 & $-0.24^{3}$ & -5.43 & -7.27 \\
\hline
\end{tabular}

Notes. $\Gamma_{4}$ and $\Gamma_{6}$ correspond to $10000 \mathrm{~K} .{ }^{(1)}$ NIST; ${ }^{(2)}$ Chang \& Tang (1990); ${ }^{(3)}$ Aldenius et al. (2007).

and velocity parameters from Anstee \& O'Mara (1995) and Barklem \& O'Mara (1997). Hereafter, these papers by Anstee, Barklem, and O'Mara are referred to as the $A B O$ theory. The quadratic Stark effect broadening constants $\Gamma_{4}$ were taken from Dimitrijevic \& Sahal-Brechot (1996), except for Mg I $4571 \AA$, with $\Gamma_{4}$ from the VALD database (Kupka et al. 1999).

\section{MgI lines in the Sun and A-type stars}

In the galactic chemical evolution studies, the stellar sample covers, as a rule, a range of metallicities of more than 3 dex. The lines Mg I 4703 and $5528 \AA$ are favorites for abundance determinations of mildly MP $([\mathrm{Fe} / \mathrm{H}]>-2)$ stars; however, these lines cannot be measured in hyper metal-poor stars, with $[\mathrm{Fe} / \mathrm{H}]<-4.5$, where one relies on the $\mathrm{Mg}$ Ib lines. One needs, therefore, to prove that the use of different $\mathrm{Mg}$ I lines does not produce a systematic shift in derived abundance between various metallicity stars. In the beginning, we checked whether the lines listed in Table 1 give consistent abundances for the Sun, when employing the most up-to-date theoretical and experimental atomic data available so far. The observed solar spectrum was taken from the Kitt Peak Solar Flux Atlas (Kurucz et al. 1984). We used the MAFAGS-OS solar model atmosphere with $T_{\text {eff }} / \log g /[\mathrm{Fe} / \mathrm{H}]=5780 / 4.44 / 0$. Microturbulence velocity was fixed at $V_{\text {mic }}=0.9 \mathrm{~km} \mathrm{~s}^{-1}$. Element abundances from individual lines were determined from line-profile fitting using the code SIU (Reetz 1991). The obtained results are presented in Table 2. We find that (i) the NLTE effects are minor for each investigated line; (ii) abundances derived from the $\mathrm{Mg} \mathrm{Ib}$ and $\mathrm{Mg}$ I $4571 \AA$ lines are consistent with the meteoritic value $\log A_{\mathrm{Mg}, \text { met }^{5}}{ }^{5}=-4.45$ (Lodders et al. 2009); and (iii) Mg I 4703 and $5528 \AA$ give significantly lower abundances, by 0.24 dex and 0.15 dex, respectively. These discrepancies can arise from the uncertainty in either $g f$-values or/and $\Gamma_{6}$ values. The influence of atmospheric inhomogeneities on derived solar $\mathrm{Mg}$ abundance was evaluated by Asplund (2005) as -0.03 dex.

To test $g f$-values, we chose the three well studied stars Sirius, Vega, and $21 \mathrm{Peg}$ (Table 3), where the MgI lines are insensitive to $\Gamma_{6}$ variation. The departures from LTE are expected to be greater in hot atmospheres than in the solar one. Thus, analysis of the MgI lines in the A-type stars also tests our NLTE method. For each star, its stellar parameters and observed equivalent widths indicated in Table 2 and used to determine element abundances were taken from a common source cited in Table 3. We find that, for each star, (i) the NLTE effects are minor for Mg I $4703 \AA$ and $5528 \AA$; (ii) abundances from these two lines are consistent within $0.02 \mathrm{dex}$, in contrast to the solar case; (iii) departures from LTE for the $\mathrm{Mg} \mathrm{Ib}$

\footnotetext{
$\log A_{\mathrm{A}}=\log N_{\mathrm{A}} / N_{\mathrm{H}}$.
} 
Table 2. Magnesium NLTE and LTE abundances $\log A_{\mathrm{Mg}}$ of the Sun and hot stars.

\begin{tabular}{|c|c|c|c|c|c|c|c|c|c|c|c|c|c|}
\hline \multirow{2}{*}{$\begin{array}{l}\lambda \\
(\AA)\end{array}$} & \multicolumn{2}{|c|}{ Sun } & \multicolumn{2}{|c|}{ HD 32115} & \multicolumn{3}{|c|}{ Vega } & \multicolumn{3}{|c|}{ Sirius } & \multicolumn{3}{|c|}{$21 \mathrm{Peg}$} \\
\hline & NLTE & LTE & NLTE & LTE & $E W^{1}$ & NLTE & LTE & $E W$ & NLTE & LTE & $E W$ & NLTE & LTE \\
\hline $\operatorname{Mg}$ I 4571 & -4.38 & -4.42 & - & - & - & - & - & - & - & - & - & - & - \\
\hline Mg I 4703 & -4.69 & -4.70 & -4.55 & -4.58 & 31 & -4.93 & -4.94 & 42 & -4.56 & -4.56 & 17 & -4.48 & -4.50 \\
\hline Mg I 5528 & -4.60 & -4.61 & -4.52 & -4.54 & 27 & -4.93 & -4.95 & 39 & -4.54 & -4.53 & 14 & -4.49 & -4.51 \\
\hline $\operatorname{Mg}$ I 5172 & -4.45 & -4.46 & -4.44 & -4.44 & 106 & -4.88 & -4.74 & 121 & -4.44 & -4.25 & 70 & -4.40 & -4.30 \\
\hline \multirow[t]{3}{*}{ Mg I 5183} & -4.45 & -4.46 & -4.44 & -4.45 & 124 & -4.84 & -4.62 & 134 & -4.44 & -4.21 & 86 & -4.35 & -4.16 \\
\hline & \multicolumn{13}{|c|}{$\Delta \log A(\operatorname{Mg}$ I $4703,5528-\operatorname{Mg}$ I 5172,5183$)$} \\
\hline & -0.19 & & -0.10 & & & -0.07 & & & -0.11 & & & -0.11 & \\
\hline
\end{tabular}

Notes. Equivalent width, $E W$, in $\mathrm{m} \AA$.

Table 3. Stellar parameters of selected stars.

\begin{tabular}{lcrrrl}
\hline \hline Object & $T_{\text {eff }}, \mathrm{K}$ & $\log g$ & {$[\mathrm{Fe} / \mathrm{H}]$} & $V_{\text {mic }}{ }^{1}$ & Ref. \\
\hline Sun & 5780 & 4.44 & 0 & 0.9 & \\
HD 32115 & 7250 & 4.20 & 0 & 2.3 & F11 \\
HD 48915 (Sirius) & 9850 & 4.30 & 0.4 & 1.8 & S13 \\
HD 84937 & 6350 & 4.09 & -2.08 & 1.7 & M11 \\
HD 103095 & 5070 & 4.69 & -1.35 & 0.8 & M07 \\
HD 122563 & 4600 & 1.60 & -2.56 & 1.95 & M11 \\
HD 172167 (Vega) & 9550 & 3.95 & -0.5 & 2.0 & P01 \\
HD 209459 (21 Peg) & 10400 & 3.55 & 0.0 & 0.5 & F09 \\
\hline
\end{tabular}

Notes. ${ }^{(1)}$ Microturbulence velocity, in $\mathrm{km} \mathrm{s}^{-1}$.

References. F09 = Fossati et al. (2009); F11 = Fossati et al. (2011); M07 = Mashonkina et al. (2007); M11 = Mashonkina et al. (2011); P01 = Przybilla et al. (2001); S13 = Sitnova et al. (2013).

lines are significant, so that the NLTE abundance correction $\Delta_{\mathrm{NLTE}}=\log \varepsilon_{\mathrm{NLTE}}-\log \varepsilon_{\mathrm{LTE}}$ ranges between -0.14 dex and -0.22 dex for different stars; and (iv) the difference in NLTE abundance between the $\mathrm{Mg} \mathrm{Ib}$ and $\mathrm{Mg}$ I 4703, $5528 \AA$ lines is smaller compared to that for the Sun. It is worth noting that it does not exceed 0.05 dex when applying $g f$-values from a single source (Chang \& Tang 1990) for all the investigated lines.

Once we return to the Sun, the abundance discrepancies found between different solar lines of $\mathrm{Mg}$ I can only be caused by the van der Waals damping treatment, and they are removed by reducing the $\Gamma_{6}(A B O)$ values by 0.3 dex and 0.2 dex for Mg I $4703 \AA$ and Mg I $5528 \AA$, respectively. The use of such corrections was justified by analysis of the $\mathrm{MgI}$ lines in the cool subdwarf star HD 103095 (Table 3). We obtained the difference $(\mathrm{Mg}$ I 4703, $5528-\mathrm{Mg} \mathrm{Ib})=-0.18 \mathrm{dex}$, when using $\Gamma_{6}(A B O)$, and a significantly lower value of +0.02 dex, with the above corrections. Table 2 presents $\mathrm{Mg}$ abundances of the late A-type star HD 32115, where Mg I $4703 \AA$ and $5528 \AA$ are less sensitive to $\Gamma_{6}$ variation. Both lines give identical abundances at $\log A_{\mathrm{Mg}}=-4.51$, and the difference $(\mathrm{Mg} \mathrm{I} 4703,5528-\mathrm{Mg} \mathrm{Ib})$ reduces to -0.07 dex, when employing the corrected $\Gamma_{6}$-values.

\section{Influence of $\mathrm{Mg}+\mathrm{H}$ collisions on the NLTE results for metal-poor stars}

The departures from LTE for Mg I grow toward lower metallicity due to the decreasing number of electrons donated by metals, which results in decreasing collision rates and also due to decreasing the UV opacity, which results in increasing photoionization rates. The largest uncertainties in NLTE results are
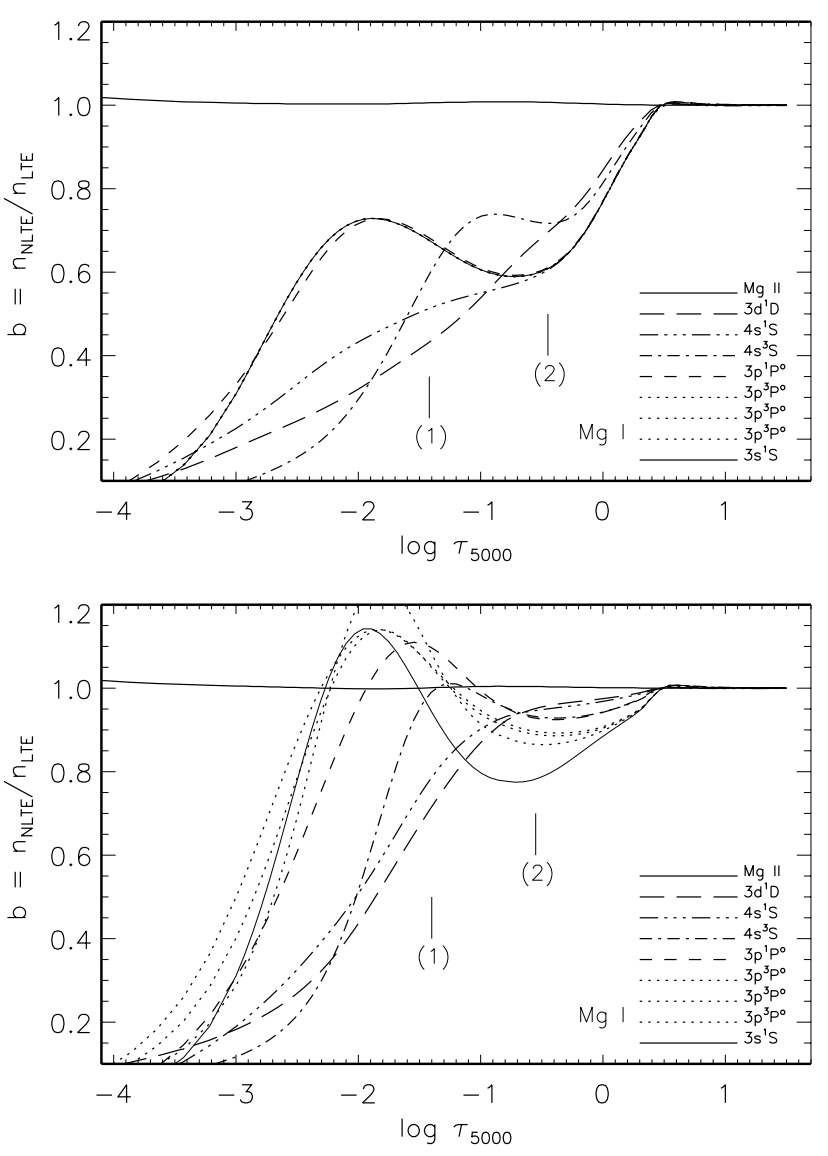

Fig. 1. Departure coefficients for selected levels of $\mathrm{Mg} \mathrm{I}$ as a function of $\log \tau_{5000}$ in the model atmosphere 4600/1.60/-2.56 from the calculations with pure electronic collisions (top panel) and with $\mathrm{H}$ I collisions taken into account following BBSGF (bottom panel). Tick marks indicate the locations of line center optical depth unity for the $\mathrm{Mg}$ I lines $5172 \AA$ ( 1 ) and $5528 \AA$ (2).

caused by the uncertainties in collisional data. Here, we apply for the first time $\mathrm{Mg}+\mathrm{H}$ collision data of Barklem et al. (2012) to calculate the SE of magnesium in two well studied VMP stars. HD 84937 represents the hot end of the stars that evolve on time scales comparable to the Galaxy lifetime. HD 122563, in contrast, is a cool giant (Table 3).

Figure 1 shows the departure coefficients $b_{i}=n_{i}^{\mathrm{NLTE}} / n_{i}^{\mathrm{LTE}}$ for the $\mathrm{Mg}$ I levels in the model atmosphere 4600/1.60/-2.56 from calculations with two different treatments of collisional rates. Here, $n_{i}^{\text {NLTE }}$ and $n_{i}^{\text {LTE }}$ are the SE and thermal (Saha-Boltzmann) number densities, respectively. In the line-formation layers, the 
A\&A 550, A28 (2013)

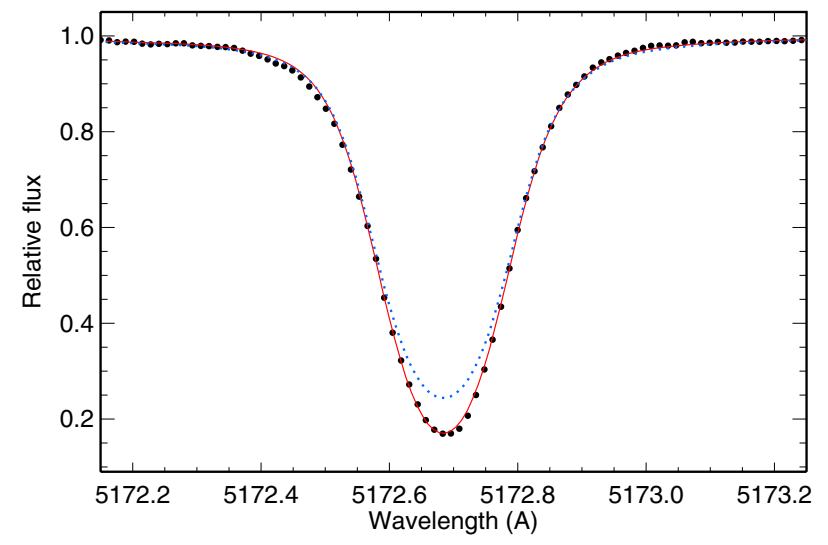

Fig. 2. Best NLTE (continuous curve) and LTE (dotted curve) fits of the Mg I $5172 \AA$ line in HD 122563 (bold dots). The NLTE calculations were performed with $\log A_{\mathrm{Mg}}=-6.95$, while the LTE ones with a 0.02 dex higher value. $\mathrm{Mg}+\mathrm{H}$ collisions are treated following BBSGF. Our theoretical flux profiles were convolved with a profile that combines instrumental broadening with a Gaussian profile and broadening by macroturbulence with a radial-tangential profile. The macroturbulence values are $4.7 \mathrm{~km} \mathrm{~s}^{-1}$ for the NLTE and $3.8 \mathrm{~km} \mathrm{~s}^{-1}$ for the LTE fits.

Mg I levels have fewer populations compared with the thermal ones, independent of collision treatment; however, the magnitude of departures from LTE is significantly smaller in the case of included $\mathrm{Mg}+\mathrm{H}$ collisions than for the case of pure electronic collisions. The main NLTE mechanism is overionization caused by the superthermal radiation of a nonlocal origin below the thresholds of the $3 \mathrm{p}^{3} \mathrm{P}^{\circ}$ and $3 \mathrm{p}^{1} \mathrm{P}^{\circ}$ levels. When taking $\mathrm{Mg}+\mathrm{H}$ collisions into account, the charge transfer processes $\mathrm{Mg}$ I $+\mathrm{HI} \leftrightarrow \mathrm{Mg}$ II $+\mathrm{H}^{-}$establish close collisional coupling of excited terms of $\mathrm{Mg}$ I to the ground state of $\mathrm{Mg}$ II and reduce their underpopulation. This effect is redistributed to the lower excitation levels via the bound-bound transitions, including the H I impact excitation and de-excitation processes.

Table 4 presents abundances determined from individual lines of $\mathrm{Mg}$ I under different assumptions for line formation. It is worth noting that results for $\mathrm{Mg}$ I $4703 \AA$ and $5528 \AA$ do not depend on the van der Waals damping treatment because both lines are weak. For each star, NLTE leads to depleted absorption in the lines at 4571,4703 , and $5528 \AA$ due to overall overionization of $\mathrm{Mg} \mathrm{I}$ in the atmospheric layers, where they form. As expected, including $\mathrm{Mg}+\mathrm{H}$ collisions leads to weaker NLTE effects compared with that for pure electronic collisions, such that $\Delta_{\text {NLTE }}$ reduces by $0.06-0.23$ dex for different lines. For HD 122563 , the NLTE effects have different signs for Mg I $4703 \AA$ and Mg I $5528 \AA$, resulting in a remarkable consistency of abundances derived from these two lines.

Despite the low $\mathrm{Mg}$ abundance, the $\mathrm{Mg}$ Ib lines are fairly strong in both VMP stars. For each line, its core forms in the layers, where the departure coefficient of the upper level $4 s^{3} \mathrm{~S}$ drops rapidly (Fig. 1) due to photon escape from the $\mathrm{Mg}$ Ib triplet lines themselves, resulting in dropping the line source function below the Planck function and enhanced absorption in the line core (Fig. 2). In contrast, in the line wings, absorption is weaker compared with the LTE case due to overall overionization in deep atmospheric layers. The combined effect is that the obtained NLTE abundances are lower than the corresponding LTE ones and lower than those from the other lines, independent of collisional rate treatment. The difference $\Delta \log \varepsilon(\mathrm{Mg}$ I 4703, 5528 $\mathrm{Mg} \mathrm{Ib})$ is less in the case of $\mathrm{Mg}+\mathrm{H}$ collisions included than in the case of pure electronic collisions and amounts to 0.09 dex for HD 84937 and 0.12 dex for HD 122563. For LTE abundances from the $\mathrm{Mg}$ Ib lines, we used $E W \mathrm{~s}$, because their profiles cannot be fitted at the LTE assumption. Despite this fact, the obtained LTE abundances are consistent within 0.05 dex with those from the other lines.

Our calculations show that inelastic collisions with $\mathrm{H}$ I atoms as treated by BBSGF produce significant thermalization effect on the SE of $\mathrm{MgI}$ in the atmospheres of VMP stars and improve $\mathrm{Mg}$ abundance determinations compared with the case of pure electronic collisions. To understand the remaining discrepancies between different lines, one probably needs to go beyond the $1 \mathrm{D}$ analysis. The models based on hydrodynamical calculations (3D models) show, on average, lower temperatures outside $\log \tau_{5000}=-1$ compared with those in the corresponding $1 \mathrm{D}$ model, for example, by $1000 \mathrm{~K}$ at $\log \tau_{5000}=-2$ in the 4858/2.2/-3 model, according to Collet et al. (2007). For VMP giants, these are exactly the layers where the line core of the $\mathrm{Mg}$ Ib lines forms.

For most atoms, the formalism of Drawin $(1968,1969)$ is still widely applied to simulate an additional source of thermalization in the atmospheres of cool stars by parametrized H I collisions. For Mg I, Barklem et al. (2012) compared in their Fig. 1 the quantum scattering rate coefficients at $T=6000 \mathrm{~K}$ with the results of the Drawin formula for five common bound-bound transitions. The Drawin/BBSGF rate ratio is close to unity for the $3 \mathrm{p}^{3} \mathrm{P}^{\circ}-4 \mathrm{~s}^{3} \mathrm{~S}$ transition, but the Drawinian rates are one to four orders of magnitude higher for the remaining transitions. However, relatively high rates compared to those for excitation were obtained by BBSGF for the charge transfer processes. We find that they are higher than the Drawinian rates for ionization from the excited levels above $3 \mathrm{p}^{1} \mathrm{P}^{\circ}$, especially from the $4 \mathrm{~s}^{1} \mathrm{~S}$ state, where the difference is about four orders of magnitude at $T=6000 \mathrm{~K}$. It is worth noting that the Drawin/BBSGF rate ratios are only weakly sensitive to temperature variation.

We employed the Drawinian rates scaled by a factor of $S_{\mathrm{H}}=$ 0.1 to determine the magnesium abundances of the investigated stars (Table 4). The changes in collisional rates result in different effects for different lines in different stars. For example, using accurate $\mathrm{Mg}+\mathrm{H}$ collision data and scaled Drawinian rates leads to very similar results for $\operatorname{Mg}$ I 4571, 4703, and $5528 \AA$ in the model 6350/4.09/-2.08. For the cool giant model, the departures from LTE are greater when applying the BBSGF rates. However, nowhere does the difference between using these two recipes exceed 0.1 dex. This is smaller than or similar to a lineto-line scatter for stellar abundance determinations including the present one.

The NLTE calculations were performed for a small grid of model atmospheres with hydrogenic collisions taken into account following BBSGF and Drawin $(1968,1969)$. The obtained NLTE corrections are presented in Table 5. Their inspection will help the user decide whether the Mg abundances determined earlier using the Drawinian rates should be revised. This depends on which spectral lines were employed in the analysis and what the range of stellar parameters was.

\section{Conclusions}

For each of four investigated A-type stars, using $g f$-values of Chang \& Tang (1990) leads to consistency within 0.05 dex between NLTE abundances from MgI $4703 \AA$, $5528 \AA$, and $\mathrm{Mg}$ Ib. The difference between different lines increases up to 0.11 dex, when employing experimental $g f$-values of Aldenius et al. (2007) for the Mg Ib lines. We recommend applying the 
Table 4. Magnesium LTE and NLTE abundances of HD 84937 and HD 122563 from calculations with different treatments of H I collisions.

\begin{tabular}{|c|c|c|c|c|c|c|c|c|}
\hline \multirow{2}{*}{$\begin{array}{l}\lambda \\
(\AA)\end{array}$} & \multicolumn{4}{|c|}{ HD 84937} & \multicolumn{4}{|c|}{ HD 122563} \\
\hline & LTE & Pure $\mathrm{e}^{-}$ & $+\mathrm{H}(\mathrm{BBSGF})^{1}$ & $+\mathrm{H}(\mathrm{D} 0.1)^{2}$ & LTE & Pure $\mathrm{e}^{-}$ & $+\mathrm{H}(\mathrm{BBSGF})$ & $+\mathrm{H}(\mathrm{D} 0.1)$ \\
\hline 4571 & -6.38 & -6.24 & -6.30 & -6.33 & -6.80 & -6.60 & -6.67 & -6.77 \\
\hline 4703 & -6.36 & -6.15 & -6.30 & -6.29 & -6.86 & -6.59 & -6.82 & -6.84 \\
\hline 5528 & -6.34 & -6.15 & -6.30 & -6.27 & -6.77 & -6.61 & -6.83 & -6.76 \\
\hline 5172 & $-6.35^{3}$ & -6.39 & -6.39 & -6.46 & $-6.87^{3}$ & -6.76 & -6.95 & -6.96 \\
\hline \multirow[t]{3}{*}{5183} & $-6.34^{3}$ & -6.39 & -6.39 & -6.46 & $-6.87^{3}$ & -6.74 & -6.94 & -6.94 \\
\hline & \multicolumn{8}{|c|}{$\Delta \log \varepsilon\left(\operatorname{Mg}\right.$ I 4703, $\left.5528-\operatorname{Mg}_{\text {I }} 5172,5183\right)$} \\
\hline & -0.01 & 0.24 & 0.09 & 0.18 & 0.05 & 0.15 & 0.12 & 0.15 \\
\hline
\end{tabular}

Notes. ${ }^{(1)} \mathrm{Mg}+\mathrm{H}$ collisions following BBSGF; (2) Drawinian rates with $S_{\mathrm{H}}=0.1$; $^{(3)}$ from $E W$.

Table 5. NLTE abundance corrections (dex) for lines of $\mathrm{Mg}$ I from calculations with $\mathrm{Mg}+\mathrm{H}$ collision rates of BBSGF and with the Drawinian rates scaled by a factor of $S_{\mathrm{H}}=0.1(\mathrm{D} 0.1)$.

\begin{tabular}{|c|c|c|c|c|c|c|c|c|c|c|c|c|}
\hline \multirow[t]{2}{*}{ Model } & \multicolumn{2}{|c|}{$3829 \AA$} & \multicolumn{2}{|c|}{$5172 \AA$} & \multicolumn{2}{|c|}{$4571 \AA$} & \multicolumn{2}{|c|}{$4703 \AA$} & \multicolumn{2}{|c|}{$5528 \AA$} & \multicolumn{2}{|c|}{$5711 \AA$} \\
\hline & BBSGF & D0.1 & BBSGF & D0.1 & BBSGF & D0.1 & BBSGF & D0.1 & BBSGF & D0.1 & BBSGF & D0.1 \\
\hline $6000 / 4.0 /-1.0$ & 0.05 & 0.02 & 0.04 & 0.02 & 0.08 & 0.05 & 0.01 & 0.04 & -0.03 & 0.04 & 0.04 & 0.07 \\
\hline $6000 / 4.0 /-2.0$ & 0.04 & 0.01 & 0.02 & -0.04 & 0.08 & 0.04 & 0.03 & 0.06 & -0.02 & 0.06 & 0.03 & 0.05 \\
\hline $6000 / 4.0 /-3.0$ & 0.08 & 0.02 & 0.05 & -0.04 & & & 0.07 & 0.07 & 0.07 & 0.08 & & \\
\hline $5811 / 4.0 /-4.5$ & 0.22 & 0.17 & 0.17 & 0.19 & & & & & & & & \\
\hline $6180 / 3.7 /-4.0$ & 0.25 & 0.19 & 0.26 & 0.21 & & & & & & & & \\
\hline $5000 / 2.0 /-1.0$ & 0.06 & 0.05 & 0.06 & 0.05 & 0.13 & 0.13 & -0.07 & 0.00 & -0.16 & -0.07 & -0.05 & 0.06 \\
\hline $5000 / 2.0 /-2.0$ & 0.08 & 0.04 & 0.03 & 0.00 & 0.16 & 0.10 & -0.02 & 0.07 & -0.12 & 0.03 & 0.03 & 0.09 \\
\hline $5000 / 2.0 /-3.0$ & 0.06 & 0.01 & -0.06 & -0.10 & 0.29 & 0.11 & 0.18 & 0.16 & 0.10 & 0.16 & & \\
\hline $5100 / 2.2 /-5.0$ & 0.40 & 0.39 & 0.34 & 0.37 & & & & & & & & \\
\hline
\end{tabular}

corrections $\Delta \log \Gamma_{6}=-0.3$ and -0.2 for $\operatorname{Mg}$ I $4703 \AA$ and $5528 \AA$, respectively, to the $A B O$ van der Waals damping constants, to remove the abundance discrepancy between different solar Mg I lines.

Inelastic collisions with $\mathrm{HI}$ atoms as treated by Barklem et al. (2012) serve as an efficient thermalizing process for the $\mathrm{SE}$ of $\mathrm{Mg} \mathrm{I}$ in the atmospheres of metal-poor stars. The use of new collisional data improves $\mathrm{Mg}$ abundance determinations for HD 84937 and HD 122563, though it does not completely remove the difference in abundance between different lines.

Acknowledgements. L.M. thanks Paul Barklem for initiating this study and providing the $\Gamma_{6}$ value for $\operatorname{Mg}$ I $4571 \AA$. This study is supported by the Ministry of education and science of Russian Federation, project 8529 and the RF President with a Leading Scientific Schools grant 3602.2012.2.

\section{References}

Aldenius, M., Tanner, J. D., Johansson, S., Lundberg, H., \& Ryan, S. G. 2007, A\&A, 461, 767

Allende Prieto, C., Asplund, M., \& Fabiani Bendicho, P. 2004, A\&A, 423, 1109 Anstee, S. D., \& O’Mara, B. J. 1995, MNRAS, 276, 859

Asplund, M. 2005, ARA\&A, 43, 481

Athay, R. G., \& Canfield, R. C. 1969, ApJ, 156, 695

Barklem, P. S., \& O'Mara, B. J. 1997, MNRAS, 290, 102

Barklem, P. S., Belyaev, A. K., Guitou, M., et al. 2011, A\&A, 530, A94

Barklem, P. S., Belyaev, A. K., Spielfiedel, A., Guitou, M., \& Feautrier, N. 2012, A\&A, 541, A80

Butler, K., \& Giddings, J. 1985, Newsletter on the analysis of astronomical spectra, No. 9, University of London

Carlsson, M., Rutten, R. J., \& Shchukina, N. G. 1992, A\&A, 253, 567

Chang, T. N., \& Tang, X. 1990, J. Quant. Spec. Radiat. Transf., 43, 207

Collet, R., Asplund, M., \& Trampedach, R. 2007, A\&A, 469, 687

Dimitrijevic, M. S., \& Sahal-Brechot, S. 1996, A\&AS, 117, 127

Drawin, H.-W. 1968, Z. Phys., 211, 404

Drawin, H. W. 1969, Z. Phys., 225, 483
Fossati, L., Ryabchikova, T., Bagnulo, S., et al. 2009, A\&A, 503, 945

Fossati, L., Ryabchikova, T., Shulyak, D. V., et al. 2011, MNRAS, 417, 495

Frebel, A., Aoki, W., Christlieb, N., et al. 2005, Nature, 434, 871

Gigas, D. 1988, A\&A, 192, 264

Gratton, R. G., Carretta, E., Eriksson, K., \& Gustafsson, B. 1999, A\&A, 350, 955

Grupp, F., Kurucz, R. L., \& Tan, K. 2009, A\&A, 503, 177

Idiart, T., \& Thévenin, F. 2000, ApJ, 541, 207

Kupka, F., Piskunov, N., Ryabchikova, T. A., Stempels, H. C., \& Weiss, W. W. 1999, A\&AS, 138, 119

Kurucz, R. L., Furenlid, I., Brault, J., \& Testerman, L. 1984, Solar flux atlas from 296 to $1300 \mathrm{~nm}$ (New Mexico: National Solar Observatory)

Lemke, M., \& Holweger, H. 1987, A\&A, 173, 375

Lodders, K., Plame, H., \& Gail, H.-P. 2009, in Landolt-Börnstein - Group VI Astronomy and Astrophysics Numerical Data and Functional Relationships in Science and Technology Volume 4B: Solar System, ed. J. E. Trümper, 44

Mashonkina, L. I., Shimanskaya, N. N., \& Sakhibullin, N. A. 1996, Astron. Rep., 40, 187

Mashonkina, L., Korn, A. J., \& Przybilla, N. 2007, A\&A, 461, 261

Mashonkina, L., Gehren, T., Shi, J.-R., Korn, A. J., \& Grupp, F. 2011, A\&A, 528, A87

Mauas, P. J., Avrett, E. H., \& Loeser, R. 1988, ApJ, 330, 1008

Merle, T., Thévenin, F., Pichon, B., \& Bigot, L. 2011, MNRAS, 418, 863

Mishenina, T. V., Soubiran, C., Kovtyukh, V. V., \& Korotin, S. A. 2004, A\&A 418, 551

Przybilla, N., Butler, K., Becker, S. R., \& Kudritzki, R. P. 2001, A\&A, 369, 1009 Przybilla, N., Nieva, M.-F., \& Butler, K. 2011, J. Phys. Conf. Ser., 328, 012015

Ralchenko, Y. A., Kramida, E., Reader, J., \& Team, N. A. 2008, NIST Atomic Spectra Database (version 3.1.5) (USA)

Reetz, J. K. 1991, Diploma Thesis, Universität München

Seaton, M. J. 1962, Atomic and Molecular Processes (New York: Academic Press)

Shulyak, D., Tsymbal, V., Ryabchikova, T., Stütz, C., \& Weiss, W. W. 2004, A\&A, 428, 993

Sitnova, T. M., Mashonkina, L. I., \& Ryabchikova, T. A. 2013, Astron. Lett., 38, in press

Steenbock, W, \& Holweger, H. 1984, A\&A, 130, 319

Tolstoy, E., Hill, V., \& Tosi, M. 2009, ARA\&A, 47, 371

Zhao, G., Butler, K., \& Gehren, T. 1998, A\&A, 333, 219 